\title{
GIANT IMPACT: ACCRETION AND EVOLUTION OF THE MOON
}

IMPLICATIONS FOR EARTH, MARS AND THE SOLAR SYSTEM AS A WHOLE'

IJ. Geiss ${ }^{1}$, M.C.E. Huber ${ }^{2}$ and A.P. Rossi ${ }^{3}$ - DOI: 10.1051/epn/2014403

口 ${ }^{1}$ International Space Science Institute, Bern

$\checkmark{ }^{2}$ Jungfraujoch Commission - Swiss Academy of Sciences

- ${ }^{3}$ Jacobs University Bremen

Our planetary system has not always been as serene as it appears to us today. Exploration of the Moon has shown that disastrous collisions and violent epochs have occurred in the early part of its history. Indeed, a collision of the Earth with another planet - the Giant Impact - is the most widely accepted theory for the origin of the Moon. Several hundred million years later, Moon and Earth received a Late Heavy Bombardment that created the large basins on the Moon and must have devastated the atmosphere and hydrosphere of the Earth. 


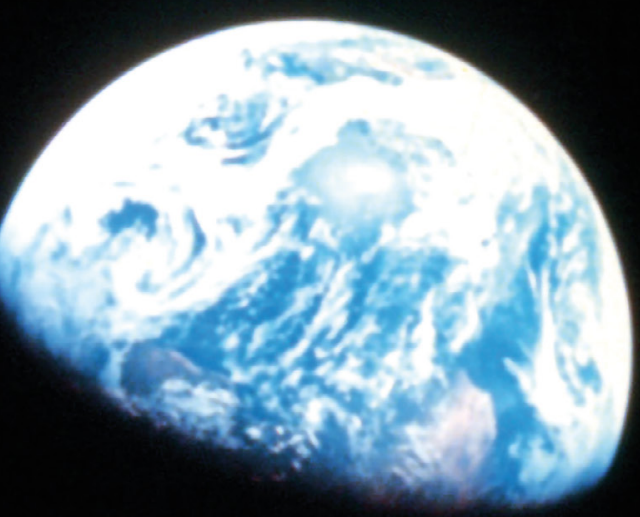

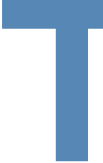

he exact place and time of such disastrous events are impossible to accurately retrace in time, but that does not mean they are unlikely. It has been proposed, for example, that the high density of Mercury is due to a giant impact as well, or that Uranus and Neptune migrated outwards, ravaging small-object populations until reaching their final positions several hundred million years after their formation. Also the existence of extra-solar "Hot Jupiters" - large planets circling close to a star - is explained by planet migration.

Obviously, the chance for life to form and survive on Earth, on Mars or on exo-planets is affected by the intensity of such violent epochs in their history.

\section{The Giant Impact}

The Giant Impact (depicted in Fig. 1) is consistent with major lunar observations, namely (i) the Moon's exceptionally large size relative to its host planet; (ii) the high angular momentum of the Earth-Moon system; (iii) the Moon's extreme depletion of volatile elements; and (iv) a differentiated global crust and mantle of the Moon that had quickly followed its delayed formation.

For the most part, the latter two findings are based on chemical analyses and age determinations of lunar rocks that had been brought back to Earth by the Apollo astronauts between 1969 and 1972 .

The Giant Impact set the initial conditions for the formation and evolution of the Moon. The impactor - sometimes called Theia - is assumed to have had at least the size of Mars and to have hit the Earth at an oblique angle. The collision produced a dense protolunar cloud. Fast accretion of the Moon from this cloud assured an effective storage of gravitational energy as heat, producing early melting. A Magma Ocean of global dimensions formed, and upon cooling, solidified into a crust ${ }^{2}$ and a mantle ${ }^{3}$ (cf. left-hand part of Fig. 2).

The Giant Impact occurred between $70 \mathrm{Ma}$ and $110 \mathrm{Ma}$ after the solar-system matter had become isolated from galactic nucleosynthesis [1]. At that time, heat and accretion were the only energy sources capable of providing the energy for melting and differentiation of a lunar-sized body ${ }^{4}$. Heating occurs near its surface and thus much heat

'Editor's Note: This special Feature is an updated summary of a paper in which J. Geiss and A.P. Rossi [The Astronomy and Astrophysics Review (2013) On the chronology of lunar origin and evolution - Implications for Earth, Mars and the Solar System as a whole, 21:68 (54 pp)] covered the development of lunar science over the past half-century and underlined the importance of understanding the Moon's history for the history of the Solar System as a whole. Detailed physical arguments, models, numerical estimates and references can be found in the review paper itself. Jo Hermans, EPN Science Editor

${ }^{2}$ The lunar crust is feldspar-rich, composed mainly of anorthite $\mathrm{CaAl}_{2} \mathrm{Si}_{2} \mathrm{O}_{8}$, with a density of $\rho=2.67 \mathrm{~g} \mathrm{~cm}^{-3}$.

The mantle is composed mainly of olivines and pyroxenes $(\mathrm{Mg}, \mathrm{Fe}) \mathrm{SiO}_{3}$ and $(\mathrm{Mg}, \mathrm{Fe})_{2} \mathrm{SiO}$ etc, with densities of $\rho \approx 3.5 \mathrm{~g} \mathrm{~cm}^{-3}$ and $\rho \approx 3.6 \mathrm{~g} \mathrm{~cm}^{-3}$, respectively. ${ }^{4}$ Decay of ${ }^{26} \mathrm{Al}$ with a half-life of $0.7 \mathrm{Ma}$ was the principal energy source for melting and differentiating asteroids very early in Solar-System history. At the time of the formation of the Moon, however, ${ }^{26} \mathrm{Al}$ had disappeared and the long-lived isotopes of $\mathrm{K}, \mathrm{U}$ and Th with half-lives between $0.7 \mathrm{Ga}$ and $14 \mathrm{Ga}$ are much too slow to produce the fast melting that is inferred from the ages of lunar crust material. 


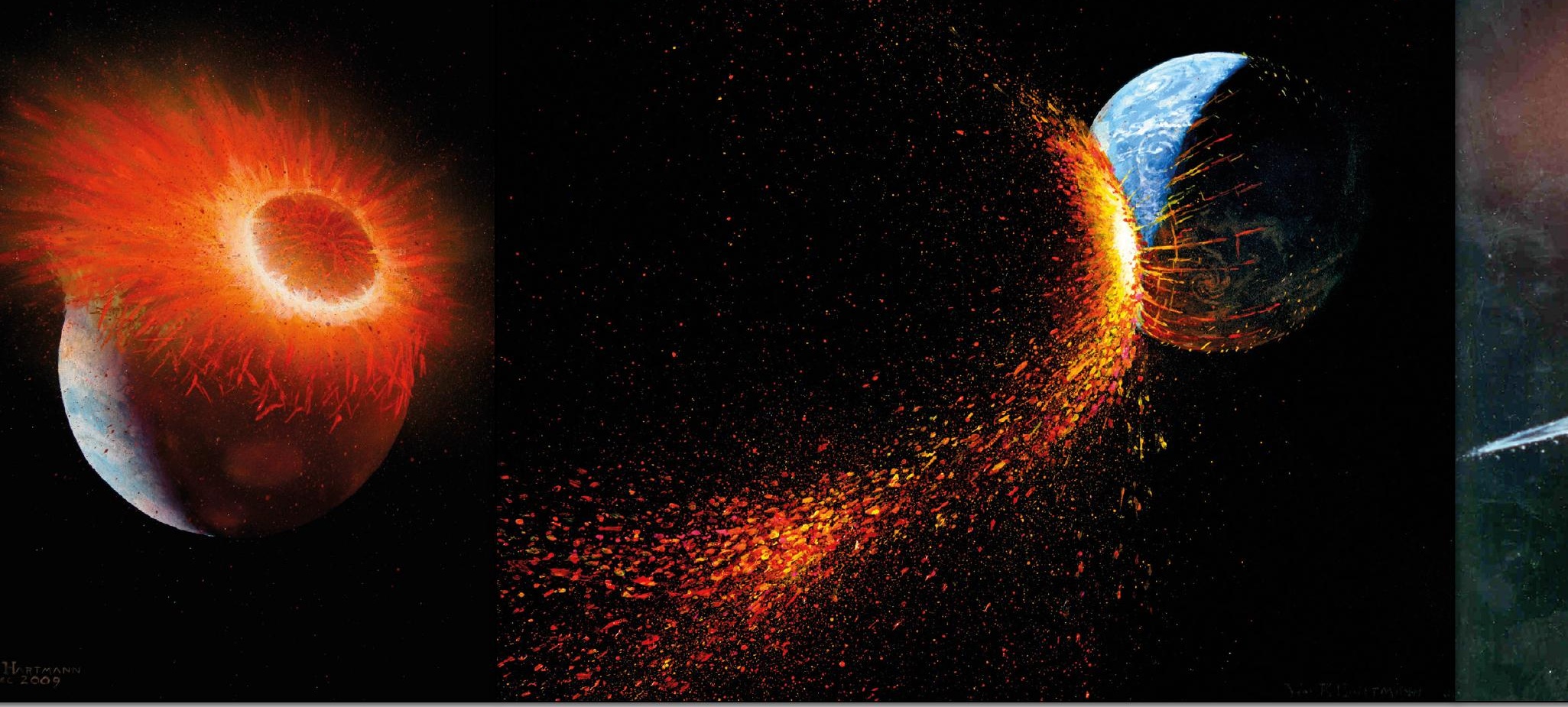

$\triangle$ FIG. 1: Origin of the Moon in a Giant Impact. Paintings by William Hartmann depicting the Earth-Moon system at $30 \mathrm{~min}$,

$5 \mathrm{~h}$ and $1000 \mathrm{a}$ respectively, after impact (@William K. Hartmann).

is lost by radiation with increasing temperature unless a very fast accretion "buries" the heat as it is produced. Accretion times as short as $\approx 10^{3}$ a are needed, and model calculations show that this is achieved at the high density existing in the protolunar cloud that had been created as a consequence of the Giant Impact.

During the formation of the Moon a poorly defined intermediate layer rich in incompatible elements called KREEP developed between crust and mantle ${ }^{5}$.

\section{The maria: basins being filled by lava}

Several $100 \mathrm{Ma}$ after the Moon's accretion, the decay of the long-lived isotopes $\left({ }^{40} \mathrm{~K},{ }^{232} \mathrm{Th},{ }^{235} \mathrm{U}\right.$ and $\left.{ }^{238} \mathrm{U}\right)$ that were concentrated in the KREEP-rich layer below the crust had generated enough internal heat to induce partial melting in the mantle. Lava extruded into large basins i.e., into the depressions that had been excavated on the Moon as part of the Late Heavy Bombardment. Upon cooling, the lava solidified into titanium-rich mare basalt ( $c f$. right-hand part of Fig. 2). This era of rock formation may actually have lasted for nearly $3 \mathrm{Ga}$, from about 3.9 Ga before the present until ca. $1 \mathrm{Ga}$ ago.

Mare basalt surfaces and other geologic units have been identified on the lunar near side by use of chemistry data from lunar orbit (i.e., from remote sensing in spectral domains ranging from $\gamma$ - and $\mathrm{X}$-rays to the infrared). How a relative timescale was established through crater counting, and how this timescale was then calibrated by radiometric dating of rocks returned from six Apollo

KREEP stands for potassium (K), rare earth elements (REE) and phosphorus $(\mathrm{P})$, which together with uranium and thorium had accumulated between crust and mantle.

${ }^{6}$ Three of the USSR Luna spacecraft returned samples that they had collected at their landing spots.

$\Delta^{17} \mathrm{O}$ is defined such as to be unaffected by mass dependent chemical or physical processes. landing regions and three Luna ${ }^{6}$ landing spots is presented in Box B. Detailed results, including geographical information are shown in Fig. 3.

The general, coherent explanation of the lunar origin and evolution sketched above is by and large complete. There remain some questions that still need to be resolved. In the following we briefly mention two such issues. The first one shows the precision of some lunar measurements, the second one also sheds light on early technological accomplishments.

\section{Constraints from isotopes:} the "Oxygen Isotope Puzzle"

The abundances of the chemical elements and their isotopes - as measured in the returned lunar samples - are generally compatible with the fractionation processes that go along with, and follow the Giant Impact origin of the Moon. But there remains an odd agreement of isotope ratios, the so-called Oxygen Isotope Puzzle, which might not $a$ priori be expected as a consequence of that scenario.

The "intrinsic" isotopic composition of an object is commonly represented as $\Delta{ }^{17} \mathrm{O}$, the excess - or deficit - of ${ }^{17} \mathrm{O}$, relative to its terrestrial abundance ${ }^{7}$. For solar-system objects, $\left|\Delta^{17} \mathrm{O}\right|$ values are very small, typically $1 \%$ or less. For Mars, for example, $\Delta^{17} \mathrm{O}=0.3 \%$ has been determined $^{8}$. Surprisingly, however, there is no significant difference in the oxygen isotopic composition between Moon and Earth.

Models have been presented indicating that this puzzle is probably not severe enough for falsifying the Giant Impact scenario. In any case, the need for explanation would be less severe if $\left|\Delta^{17} \mathrm{O}\right|$ of Theia was smaller than the Martian value of $0.3 \%$. This is quite possible, since Theia, by definition, crossed the Earth orbit during the later phase of planet accretion, while Mars accreted its matter farther away from Earth. So it is quite possible that Theia's $\left|\Delta^{17} \mathrm{O}\right|$ was very small. 


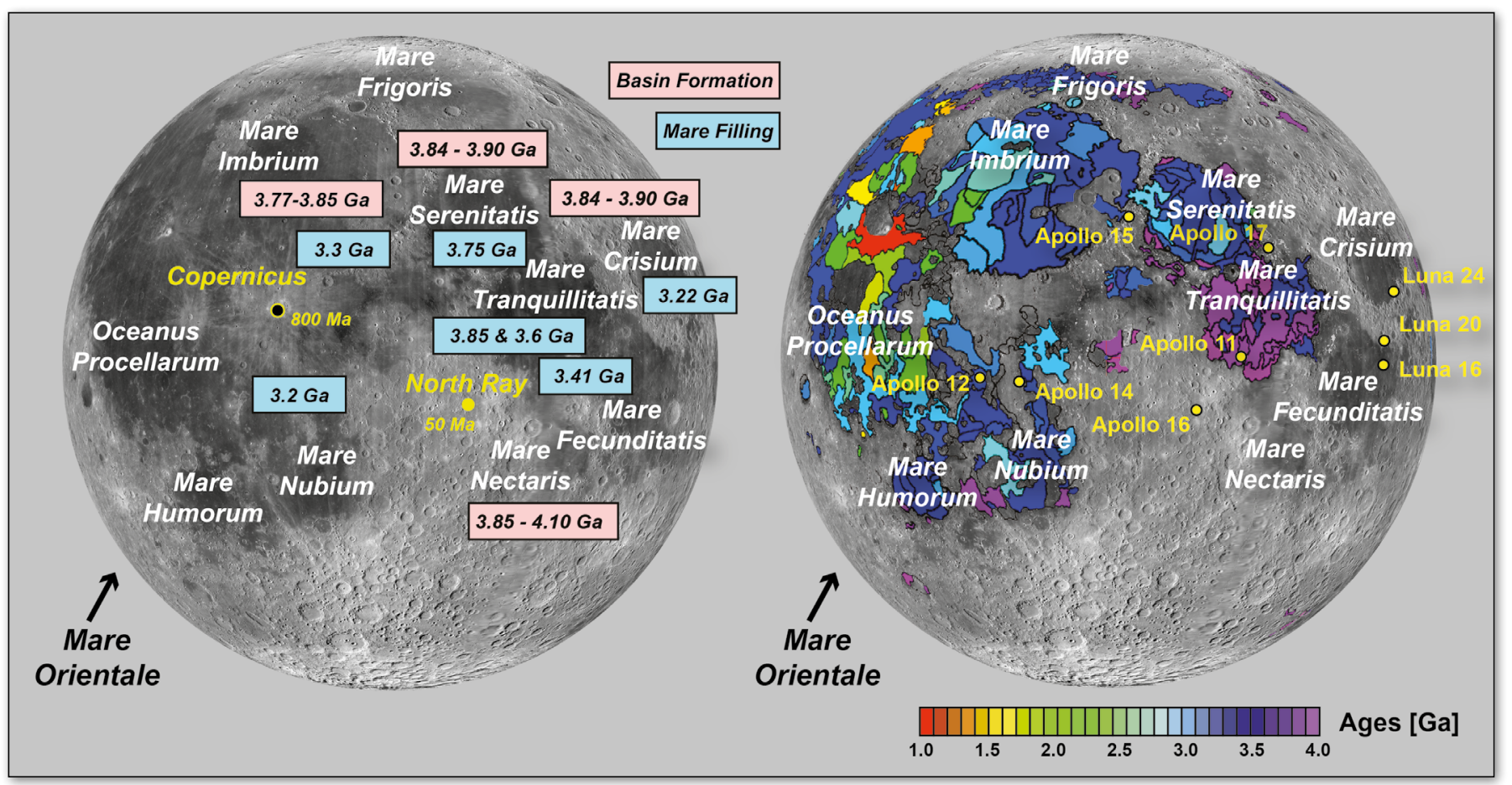

because the meteorite ejection ages coincide with the crater frequency age of that crater as calibrated with a theoretical Mars Production Function. Both ages cluster around $3 \mathrm{Ma}$. The expected production rate on Mars for Craters with a diameter D $>55 \mathrm{~km}$ is less than one in $10 \mathrm{Ma}$. Thus having a competitor for the Mojave Crater as source for the 3-Ma-Shergottites is rather unlikely. In fact, the ample ejection of meteorites from the young Mojave Crater might be the reason why in our epoch there are more Martian meteorites than lunar ones!

Clustering of exposure ages is observed not only for Shergottites, but also for other meteorite classes, suggesting that crater production rates are likely to vary on timescales of tens of Ma. For older surfaces such variations will be evened out when observing Cumulative Crater Frequencies.
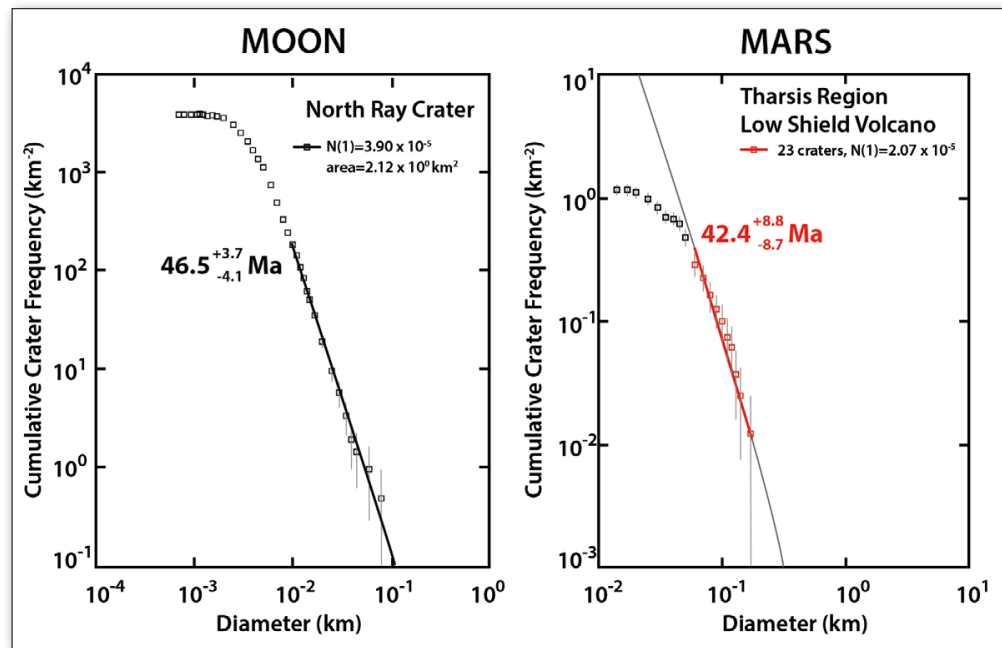

\section{The Late Heavy Bombardment}

Radiometric dating revealed that four of the prominent large lunar basins were created during a relatively short time interval, about $4.1 \mathrm{Ga}$ to $3.8 \mathrm{Ga}$ before present (cf. Fig. 3 and the illustration in Box A). When stratigraphic observations showed that many of the other large basins on the near side of the Moon were also excavated during or close to this period, it was realised that the Moon had received a Late Heavy Bombardment (LHB), about 0.5 Ga to $1.6 \mathrm{Ga}$ after the Moon had been accreted. Such a late clustering of major impacts must have been caused by a special phase in the history of the solar system, separate from the main accretion period. Was there a major perturbation in the planetary system so late in its history, and what could have been its cause?

Three discoveries, the lunar LHB, Trans-Neptunian objects, and Jupiter-sized Exoplanets unexpectedly close to their central stars[6] have renewed interest in planet

$\triangle$ FIG. 3 Two images of the near side of the Moon with chronological information. (left) Radiometric ages of four large basins (purple) and basalt samples from six mare areas (blue) derived from analyses of samples collected in the Apollo landing areas and the Luna landing spots (both of them are marked on the right-hand image). (right) (rater frequency ages of mare areas from high-resolution crater counting and mapping. The youngest mare areas are in the Oceanus Procellarum to the West of Mare Imbrium; they correspond to mare areas with a high KREEP content.

४FIG. 4 Crater frequency ages for the lunar North Ray Crater [2] and a young volcanic area on Mars.[3] Images used were from the NASA Lunar and Mars Reconnaissance Orbiters, respectively. The Crater Frequency ages given in these plots were calculated by using the theoretical Production-Function and Chronology-Function given by Ivanov [4] (see Box B). 
migration, and led to the development of Nice Models ${ }^{13}$ for explaining late heavy bombardment epochs by planet rearrangement well after the birth of the Solar System.

The classical Nice model assumes planets to have been accreted from the proto-planetary disc more closely together than we find them today. After dissipation of the gaseous portion of the solar nebula, the planets slowly migrated, due to their interacting with the remaining planetesimals. Saturn moved away from Jupiter, increasing the ratio between the periods of the two planets $\left(P_{\mathrm{S}}\right.$ and $\left.P_{\mathrm{J}}\right)$, until they got into the $1: 2$ resonance at $P_{\mathrm{j}} / P_{\mathrm{S}}=1 / 2$ (today, we have $\left.P_{\mathrm{j}} / P_{\mathrm{S}}=1 / 2.48\right)$. The orbit of Saturn became very eccentric, causing close encounters among large planets and "a massive delivery of planetesimals to the inner solar system" that could have caused the lunar LHB.[7]

For comparisons with specific Nice-model predictions, the lunar LHB would have to be better defined than it is today. The Orientale basin is the youngest among the major lunar basins and may mark the end of the lunar LHB. Its beginning is difficult to measure and even to define. Determining instead the declining phase of the bombardment by measuring the number of basin-forming impacts in a given time interval near $4 \mathrm{Ga}$ would be easier and could significantly constrain theories of LHB origin. Geiss \& Rossi ${ }^{1}$ suggest that two rover/samplereturn missions would do the job, one sampling the blanket of the Orientale basin and the other exploring a region to the Southeast of Mare Nectaris ${ }^{14}$.

The heavy bombardment of the Moon about $4.1 \mathrm{Ga}$ to 3.8 Ga ago must have hit the Earth as well, causing lasting devastation of our atmosphere and hydrosphere ${ }^{15}$. Quantitative comparison of the effects of bombardment of Moon and Earth depends on the origin of the impactor. For objects arriving with high energy from, e.g., the region beyond Uranus, impact energies on the Earth are only modestly increased, and the Moon and Earth ratio of impact rates are nearly proportional to their geometrical cross sections (1: 13.4). On the other hand, for objects coming from the inner edge of the asteroid belt, focussing and acceleration by the terrestrial gravitational field strongly increases the relative flux and also the impacting energy hitting the Earth.

Remnants of basins produced on Earth during the Archean and Proterozoic epochs have been wiped out by plate tectonics. However, the distribution of spherule beds, interpreted as ejecta from large impacts, indicate

\footnotetext{
${ }^{13}$ Nice models were first developed in Nice (France).

${ }^{14}$ Following the delivery to the lunar surface of the Chinese rover Chang'e 3 in December 2013, a sample return mission, Chang'e 5 is scheduled for 2017.

${ }^{15}$ The energy of the Imbrium impact on the Moon was roughly a thousand times higher than the energy of the Chixculub impact on Earth that caused the mass extinction at the K/T boundary.
}

\section{BOX A:TIMESCALES AND CHRONOLOGIES[9]}

On Earth, a global relation was found in the $19^{\text {th }}$ century between fossil species and sedimentary rock strata, establishing a relative chronology from the Cambrian to the present. In the $20^{\text {th }}$ century this chronology was calibrated by radiometric dating and extended deep into the Precambrian. On the Moon, where there are no fossils, relative timescales are based on the observed impact crater frequencies. Crater count ages have now been determined for a large portion of the lunar surface and calibrated with radiometric and some exposure ages of lunar samples returned from six Apollo landing areas and three Luna landing spots. Even from the rather limited geographical coverage available after the Apollo and Luna missions, it was concluded that the crater production rate decreased strongly for ages larger than $3.2 \mathrm{Ga}$ and remained approximately constant afterwards. By approximating the crater production rate by a constant plus an exponential, Neukum, Ivanov and Hartmann[10] introduced the Cumulative Crater Frequency Timescale shown in the illustration below; this has since been the standard. The timescale is well established only in the time interval $4 \mathrm{Ga}$ to $3 \mathrm{Ga}$. It cannot be much extended beyond $4 \mathrm{Ga}$, because there the crater frequency is approaching saturation. Samples from the young areas to the West of Mare Imbrium (cf. Fig. 3) could close the gap in the calibrated timescale from $3 \mathrm{Ga}$ to $1 \mathrm{Ga}$. Completing the lunar crater count timescale is of foremost importance, because it will remain indispensable for quite some time, as it can help for transferring absolute timescales to Mars and other solar system bodies.

VFIG.5:Timescale of events on the Moon, traced by the relative scale established by crater counting, which was calibrated by radiometry of lunar rock samples. Red, blue and purple data points represent the Late Heavy Bombardment, the maria and relatively young craters, respectively.

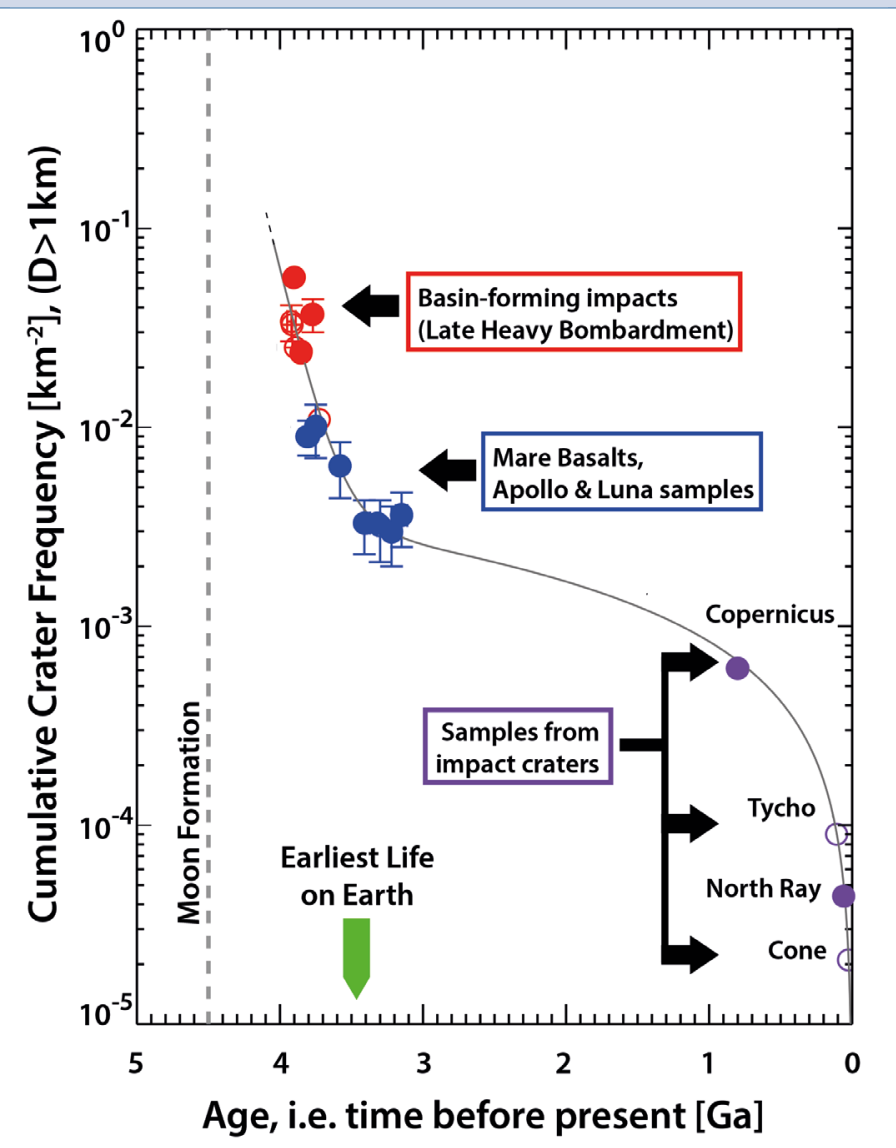


that basin forming impacts on Earth lasted to $3 \mathrm{Ga}$ before the present and beyond. [8] The lunar LHB ended earlier, as inferred from the crater frequency curve in the illustration of Box A or from the estimated age of the Orientale basin.

The earliest, well-established traces of life appeared about $3.5 \mathrm{Ga}$ ago. The analysis of spherule beds implies, however, that the epoch of heavy bombardment on Earth had not yet ended at that time. Further data on the LHB and post-LHB epochs on Moon, Earth and Mars as well as on early traces of life on Earth might tell us whether a heavy bombardment had delayed the creation of life on our planet, whether all life was extinguished that had existed before, or whether a primitive life form managed somehow to survive the epoch of heavy bombardment.

\section{BOX B: AGE DETERMINATION}

Radiometric Ages are determined by radioactive dating. This technique is based on a comparison between the observed abundance of naturally occurring radioactive isotopes and their decay products, by use of the known decay rates.

Cosmic Ray Exposure Ages are based on the following model: a collision excavates a rock that was buried and screened from cosmic rays and ejects it into an orbit around the Sun, where it is fully exposed to cosmic rays. Eventually, the orbiting rock will collide with the Earth, where cosmic-ray exposure will virtually cease. The time of exposure to cosmic rays is determined from nuclides produced by cosmic-ray-induced nuclear reactions.

An exposure age $\left(T_{\text {ExP }}\right)$ is calculated from the abundances of a pair of a stable $\left(N_{\mathrm{ST}}\right)$ and a radioactive $\left(N_{\mathrm{RAD}}\right)$ cosmic-ray-produced nuclide, the corresponding production rates $P_{\mathrm{ST}}$ and $P_{\mathrm{RAD}}$, and the decay constant $\lambda$ of the radioactive nuclide. If $P_{\mathrm{ST}}$ and $P_{\mathrm{RAD}}$ were constant over the time interval $T_{\text {EXP }}$ to $T_{0}$, then for the simple case of $\lambda T_{0}=0$ and $\lambda T_{\text {EXP }}>>1$, the exposure age is given by

$$
T_{\mathrm{EXP}}=\frac{1}{\lambda} \frac{P_{\mathrm{RAD}}}{P_{\mathrm{ST}}} \frac{N_{\mathrm{ST}}}{N_{\mathrm{RAD}}}
$$

This relation is valid only if the irradiation conditions (cosmic-ray intensity and energy spectrum, heliospheric modulation, shielding by atmosphere or overlying solid material) were constant from $T_{\mathrm{EXP}}$ to $T_{0}$. These conditions are usually met when calculating the time of ejection of a meteorite from an asteroid, Mars or Moon.

Crater Count Ages are defined as the Cumulative-Crater-Frequency $\left[\mathrm{km}^{-2}\right]=$ Production-Function (D) $x$ Chronology-Function $(\mathrm{T})\left[\mathrm{km}^{-2}\right]$. Because it is possible to separate the variables, the data can be presented in two types of plots:

Cumulative-Crater-Frequency versus Age (see graph in BOX A) and Cumulative-Crater-Frequency versus $D$, i.e., the lower integration limit for the crater diameter.

The slope of the plot (cf. Fig. 4) determines the age of the cratered area. This must, however, be calibrated by radiometric or exposure ages of rocks, determined in the laboratory.

\section{About the Authors}

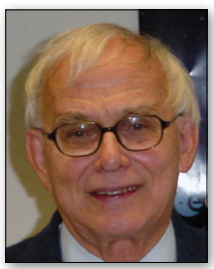

Johannes Geiss is Honorary Director and was Executive Director (1995-2002) of the International Space Science Institute in Bern. He was Professor of Physics at the University of Bern (1960-1991), Associate Professor at the Marine Laboratory of the University of Miami (1958-1959) and Research Associate at the Enrico Fermi Institute, University of Chicago (1955-1956). He is a Foreign Associate of the US National Academy of Sciences and a Member of the Academia Europaea. He received the NASA Medal for Exceptional Scientific Achievement, the Albert Einstein Medal of the Einstein Association in Bern, and the Bowie Medal of the American Geophysical Union.

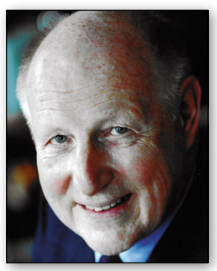

Martin C.E. Huber was Adjunct Professor at ETH Zurich, Head of ESA's Space Science Department and President of EPS (2004-2005). He was closely associated with Europhysics Letters (now EPL) and is a member of the Editorial Board of The Astronomy and Astrophysics Review. He is an Associate of Harvard College Observatory, a Member of the Academia Europaea and immediate past President of the Kommission für die Hochalpine Forschungsstation Jungfraujoch of the Swiss Academy of Sciences (SCNAT).

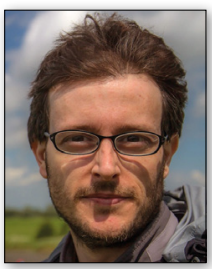

Angelo Pio Rossi is a planetary scientist based at Jacobs University Bremen. $\mathrm{He}$ is a geologist by background and works on Earth and Planetary Remote Sensing and Comparative Planetology. $\mathrm{He}$ is a Co-I of the High Resolution Stereo Camera on board of ESA's Mars Express and a member of the Editorial Boards of Planetary and Space Science and EGU Solid Earth. Previously he was a Research Fellow in ESA's Science Directorate and Staff Scientist in the International Space Science Institute.

\section{References}

[1] see A.N. Halliday, Phil Trans R Soc A 366, 4163 (2008).

[2] H. Hiesinger, C.H. Van der Bogert, J.H. Pasckert et al., J Geophys Res 117, EOOH10 (2010).

[3] F. Jagert, E. Hauber, Geoinf 2012 (2), 177 (2012).

[4] B.A. Ivanov, Space Sci Rev 96, 87 (2001).

[5] S.C. Werner, A. Ody, F. Poulet, Science 343, 1343 (2014).

[6] M. Mayor \& D. Queloz, Nature 378, 355 (1995).

[7] R. Gomes, H.F. Levison, K. Tsiganis, A. Morbidelli, Nature 435 466 (2005).

[8] B.C. Johnson and H.J. Melosh, Nature 485, 75 (2012); W.F. Bottke, D. Vokrouhlicky, D. Minton et al., Nature 485, 78 (2012).

[9] R. Kallenbach, J. Geiss and W.K. Hartmann, Space Sci Ser ISSI (SSSI) 12 (2001).

[10] G. Neukum, B.A. Ivanov, W.K. Hartmann, Space Sci Rev 96, 55 (2001), and SSSI 12, 55 (2001). 